\title{
Simulations of interference effects in gated two-dimensional ballistic electron systems
}

Jauho, Antti-Pekka; Pichugin, K.N.; Sadreev, A.F.

Published in:

Physical Review B

Link to article, DOI:

10.1103/PhysRevB.60.8191

Publication date:

1999

Document Version

Publisher's PDF, also known as Version of record

Link back to DTU Orbit

Citation (APA):

Jauho, A-P., Pichugin, K. N., \& Sadreev, A. F. (1999). Simulations of interference effects in gated twodimensional ballistic electron systems. Physical Review B, 60(11), 8191-8198.

https://doi.org/10.1103/PhysRevB.60.8191

\section{General rights}

Copyright and moral rights for the publications made accessible in the public portal are retained by the authors and/or other copyright owners and it is a condition of accessing publications that users recognise and abide by the legal requirements associated with these rights.

- Users may download and print one copy of any publication from the public portal for the purpose of private study or research.

- You may not further distribute the material or use it for any profit-making activity or commercial gain

- You may freely distribute the URL identifying the publication in the public portal

If you believe that this document breaches copyright please contact us providing details, and we will remove access to the work immediately and investigate your claim. 


\title{
Simulations of interference effects in gated two-dimensional ballistic electron systems
}

\author{
Antti-Pekka Jauho \\ Mikroelektronik Centret, Technical University of Denmark, Bldg 345east, DK-2800 Lyngby, Denmark \\ Konstantin N. Pichugin \\ Kirensky Institute of Physics, 660036 Krasnoyarsk, Russia \\ and Institute of Physics, Academy of Sciences of the Czech Republic, Cukrovarnicka 10, 16200 Prague, Czech Republic \\ Almas F. Sadreev \\ Kirensky Institute of Physics, 660036 Krasnoyarsk, Russia \\ and Department of Physics, Åbo Akademi, SF-20500 Turku, Finland
}

(Received 19 January 1999)

\begin{abstract}
We present detailed simulations addressing recent electronic interference experiments, where a metallic gate is used to locally modify the Fermi wavelength of the charge carriers. Our numerical calculations are based on a solution of the one-particle Schrödinger equation for a realistic model of the actual sample geometry, including a Poisson equation-based determination of the potential due to the gate. The conductance is determined with the multiprobe Landauer-Büttiker formula, and in general we find conductance vs gate voltage characteristics, which closely resemble the experimental traces. A detailed examination based on quantummechanical streamlines suggests that the simple one-dimensional semiclassical model often used to describe the experiments has only a limited range of validity, and that certain "unexpected" periodicities should not be assigned any particular significance, they arise due to the complicated multiple scattering processes occurring in certain sample geometries. [S0163-1829(99)00435-X]
\end{abstract}

\section{INTRODUCTION}

Recent years have witnessed many experimental and theoretical advances addressing the physical properties of mesoscopic samples, i.e., structures where the phase of the electronic wave function directly affects the measurable properties. ${ }^{1}$ A standard way to modify the phase of the wave function is to use external magnetic fields: electrons traversing the sample along a given path will accumulate a phase $\phi=(e / h) \int \mathbf{A} \cdot d \mathbf{l}$, and thus give rise to interference phenomena, such as Aharonov-Bohm oscillations. Recently Yacoby et $a l^{2,3}$ demonstrated another way of affecting the phase: a biased metallic gate, placed above the two-dimensional electron gas, will change the electron density (or, equivalently, the local Fermi wave length) underneath it, and thus introduce a phase difference between electronic paths that pass under the gate, and those that do not. In the first experiment ${ }^{2}$ the amplitude of the interference signal was used to extract the energy, or temperature, dependence of the dephasing length in a ballistic system. The experimental findings allowed a detailed comparison with theoretical predictions ${ }^{4}$ thus underscoring the importance of this technique. The second experiment ${ }^{3}$ was the first demonstration of a double-slit interference experiment in a solid state system. Both of these experiments were analyzed with the help of the following simple model. Assuming that the charge density is constant under the gate (but different than elsewhere in the sample), Refs. 2 and 3 find that the phase difference $\Delta \phi$ of two representative one-dimensional paths is given by $\Delta \phi=w\left(k_{F}\right.$ $\left.-k_{F}^{\prime}\right)=w k_{F}\left[1-\sqrt{1-\left(V_{g} / V_{\text {dep }}\right)}\right]$. [Here $w$ is the width of the gate, $k_{F}$ and $k_{F}^{\prime}$ are the Fermi momenta of the unmodified two-dimensional electron gas (2DEG) and the 2DEG under the gate, respectively, and $V_{\text {dep }}$ is the gate voltage required to entirely deplete the region under the gate. $\left.{ }^{5}\right]$ Indeed, the measured conductances have a periodic component that essentially scales with the square root of the gate voltage. In spite of this qualitative agreement, some outstanding problems remain. In particular, a Fourier analysis of the periodic signal of the double-slit experiment ${ }^{3}$ contained an unexpected lowfrequency component, approximately at half of the frequency of the main feature. Yacoby et al. ${ }^{3}$ tested a number of plausible causes for this behavior (geometric effects, higher electronic subbands, and spin-orbit interactions), but concluded that none of these could satisfactorily explain the observations, which thus remained a puzzle.

The semiclassical picture discussed above is very persuasive, and indeed it has been used in a large number of other contexts as well. The geometries studied by Yacoby et al. ${ }^{2,3}$ are quite complicated, and the possibility remains that an analysis based on one-dimensional straight paths may miss some essential features. To the best of our knowledge, these structures have not been analyzed in terms of a full solution of the appropriate Schrödinger equation, and the purpose of this paper is to report such a study, the aim being the resolution of the problems encountered in the interpretation of the second experiment. ${ }^{3}$ Our paper can be seen as a natural extension of several recent works reporting detailed solutions of the Schrödinger equation for experimentally relevant semiconductor nanostructures. ${ }^{6}$ The resulting wave functions often display a very rich structure and even surprising physics, and as an example we mention vortices around nodal points. ${ }^{7}$ The new ingredient in our paper is that, in addition to focusing on two recent experimental geometries, we include the effect of the phase-modulating gate (PMG) on the 


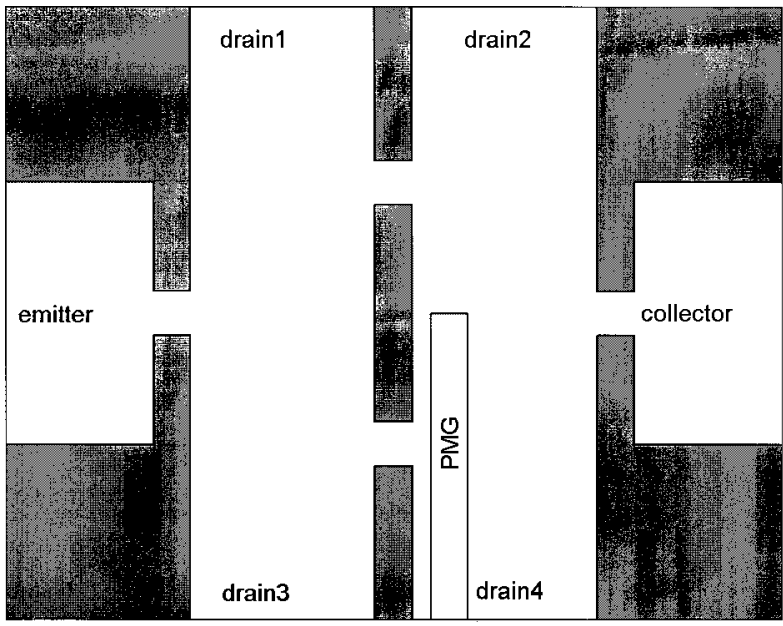

FIG. 1. Schematic representation of the six-terminal structure. The boundaries of the structure and the double slit are shown as shaded areas; they are modeled as hard walls. The lithographic placement for the phase-modulating metallic gate (PMG) which is deposited on the surface of the sample at height $h$ above the 2DEG (typically $h \simeq 100 \AA$ ) is indicated as a white rectangular area. The corresponding potential is shown in Fig. 2.

potential landscape in which the electrons move. Further, we generate stream-lines from the probability-current flow; this allows us to quantify the role played by the various paths contributing to conductance. Our main conclusions are as follows. The effects of the gate can be felt in large regions in the sample, and, in particular, in the double-slit geometry the PMG also affects the slit region. It turns out that a description based on a few characteristic paths works reasonably well in the geometry of the earlier experiment; ${ }^{2}$ this is not at all the case for the double slit geometry. In general, we find that the resulting conductance vs gate-voltage curves are very sensitive to the details of the geometry. In particular, the shape of the emitter and collector quantum point contacts is found to play an important role. For certain parameters the simulated conductance curves resemble closely their experimental counterparts, yet in other cases, with nominally small changes in the parameter values, even the qualitative appearance can change drastically. The numerical evidence suggests that one should not assign major importance to specific features in the Fourier transforms of the periodic conductance curves: they may just reflect some details of the sample geometry and do not allow a simple semiclassical interpretation.

We should note an important limitation of our numerical calculations. If we express all energies in terms of a parameter $E_{0} \equiv \hbar^{2} /\left(2 m^{*} d^{2}\right)$, where $d$ is the width of the injecting electrode, the experiments typically involve energies of the order of 20000-30000 (we estimate $d$ from published electron micrographs). Our numerical resources do not allow energies much higher than 5000, i.e., one fourth to one sixth of the experiments. As a consequence, the range of gate voltages we can study is somewhat smaller than what can be achieved experimentally, but nevertheless we believe that our simulations have direct relevance on the reported measurements.

This paper is organized as follows. In Sec. II, we describe the method of calculation, Sec. III is devoted to the analysis of the first experiment, and Sec. IV presents our results for the double-slit geometry.

\section{THE MODEL}

The generic structure considered in this work is shown in Fig. 1. It consists of the emitter, collector, phase-modulating gate (PMG), and (possibly) the double slit (DS). The emitter and collector are modeled as quantum point contacts. An important role is played by the four base contacts: electrons scattering off from the DS or PMG, and not making it to the collector leave the device via these contacts and do not contribute to further interference patterns. In the simulations these ideal base contacts are represented by open (Neumann) boundary conditions. In effect, then, we are considering a six-terminal geometry (emitter, collector, and four base contacts). The boundaries defining the structure are taken as hard walls, and the potential describing the PMG is described below. Our numerical method for the solution of the Schrödinger equation is quite standard, and here we only give those special features that are necessary for understanding the computed results, given in several figures below. The incident wave function in the $n$th transverse channel is given by

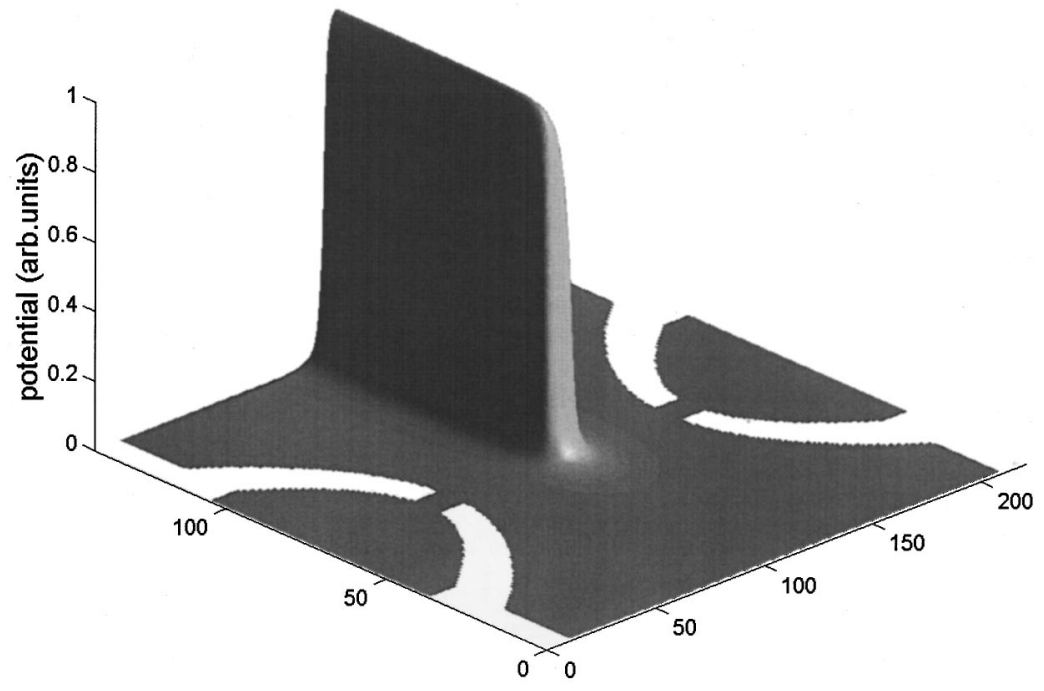

FIG. 2. The characteristic shape of the electrostatic potential due to the phase-modulating gate. Also are shown curved emitter and collector contacts as used in the experiment of Ref. 2. 

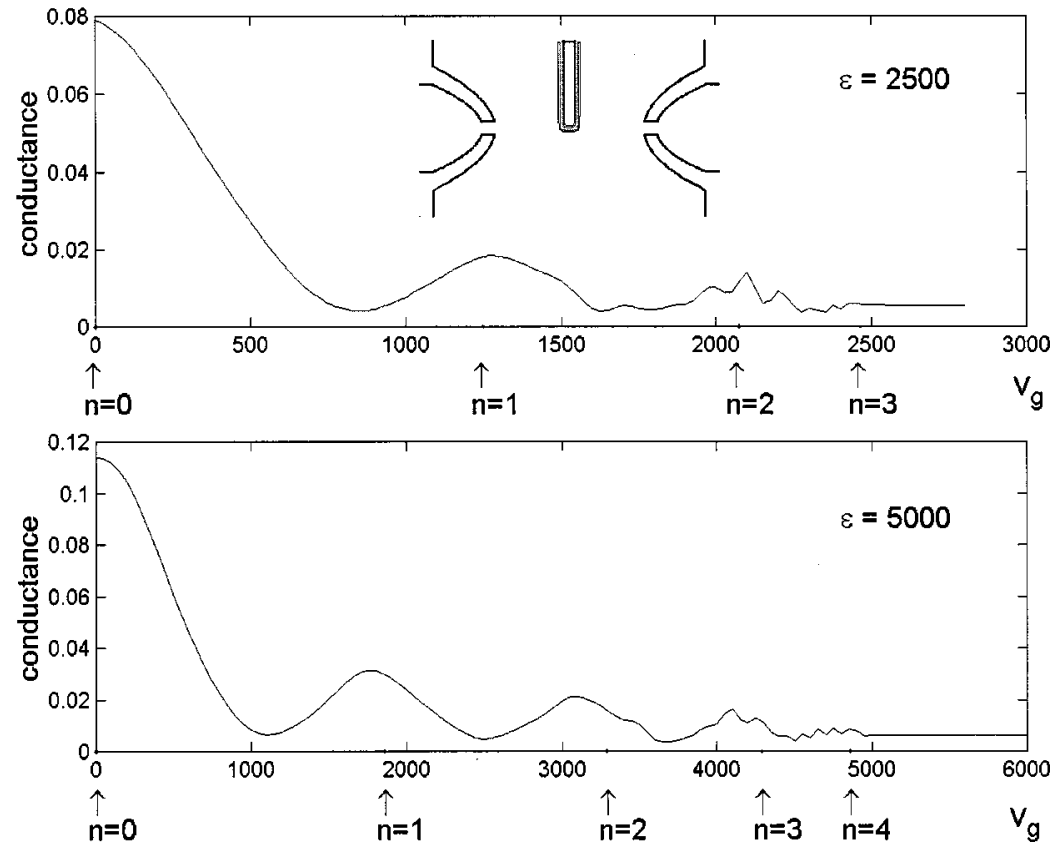

FIG. 3. Conductance vs gate voltage for the device of Fig. 2 (shown as inset) for initial energies $\epsilon=2500$ (top) and $\epsilon=5000$ (bottom). The predictions of the semiclassical formula for conductance maxima are shown as arrows.

$$
\psi_{i n, n}(x, y)=\sin (\pi n x) e^{-i k_{n} y}+\sum_{m} r_{n m} \sin (\pi m x) e^{i k_{m} y},
$$

where the sine-functions are the transverse eigenfunctions of the injecting electrode of unit width $(d=1), r_{n m}$ are the corresponding reflection coefficients, and the wave vectors $k_{n}$ are defined via

$$
\epsilon=k_{n}^{2}+\pi^{2} n^{2},
$$

where the energy $\epsilon$ is given in units of $E_{0}$ defined above, and $k_{n}$ is in units of $1 / d$. Analogously, the collector wave function is expressed as

$$
\psi_{\text {out }, n}(x, y)=\sum_{m} t_{n m} \sin (\pi m x) e^{-i k_{m} y},
$$

where $t_{n m}$ is the transmission coefficient from mode $n$ to mode $m$, and the collector is assumed to have the same width as the emitter. Finally, the boundary conditions at the $s$ th base contact (of width $L_{s}$ ) are specified by

$$
\psi_{s, n}(x, y)=\sum_{m} t_{s, n m} \frac{1}{\sqrt{L_{s}}} \sin \left(\pi m \frac{y-y_{s}}{L_{s}}\right) e^{i k_{s, m}\left(x-x_{s}\right)} .
$$

Here, $x_{s}, y_{s}$ are the coordinates of the walls defining the base contact $s$, and $k_{s, m}^{2}=\epsilon-\pi^{2} m^{2} / L_{s}^{2}$.

The Hamiltonian in a tight-binding representation for the two-dimensional electron system is

$$
\hat{H}=-t\left(c_{i, j}^{\dagger} c_{i, j+1}+c_{i, j}^{\dagger} c_{i+1, j}+\text { H.c. }\right)+V_{i, j} c_{i, j}^{\dagger} c_{i, j},
$$

where $V_{i j}$ is the electrostatic potential due to the PMG. We use the transfer matrix method as formulated in Ref. 8 to compute the various transmission and reflection coefficients (and hence the conductances). ${ }^{9}$

A compact theory for the electrostatic potential caused by metallic gates on the surface of the heterostructure has been developed by Davies et al. ${ }^{10}$ for a number of different physical assumptions (pinned surface, frozen surface, linear screening, etc.). Following their analysis we choose ${ }^{11}$ (here $h$ is the distance between the gate and the 2DEG)

$$
V(x, y, z=h)=\frac{V_{g}}{\pi}[f(x, y-w / 2, h)-f(x, y+w / 2, h)],
$$

where

$$
\begin{gathered}
f(x, y, h)=\arctan \left[\frac{h}{R-x-y}\right], \\
R=\sqrt{x^{2}+y^{2}+h^{2}} .
\end{gathered}
$$

A typical potential profile is shown in Fig. 2.

\section{THE EFFECT OF PHASE-MODULATING GATE}

We first consider the experiment reported in Ref. 2. The aim there was to study dephasing due to electron-electron collisions in a ballistic sample, and the phase modulating gate was introduced to generate an interference signal: the amplitude of the oscillatory component of the conductance is a direct measure of the phase-coherent part of the electrons, and thus allows one to extract the phase-breaking rate as a function of injection energy or temperature, and compare it to theoretical predictions. ${ }^{4}$ The actual sample had two 'semiinfinite' gates, however only one of them was activated and we therefore model the sample geometry as shown in the inset of Fig. 3, with the phase-modulating gate-potential given by Fig. 2 (the infinitely high-potential barriers defining the emitter/collector contacts are white). Let us first construct an analytic estimate for the expected behavior. Fixing the coordinates so that the PMG runs parallel to the $x$ axis, and that the electron moves along the $y$ axis, the semiclassical formula for the phase accumulated under the PMG is 
$\varepsilon=2500, v_{g}=0$

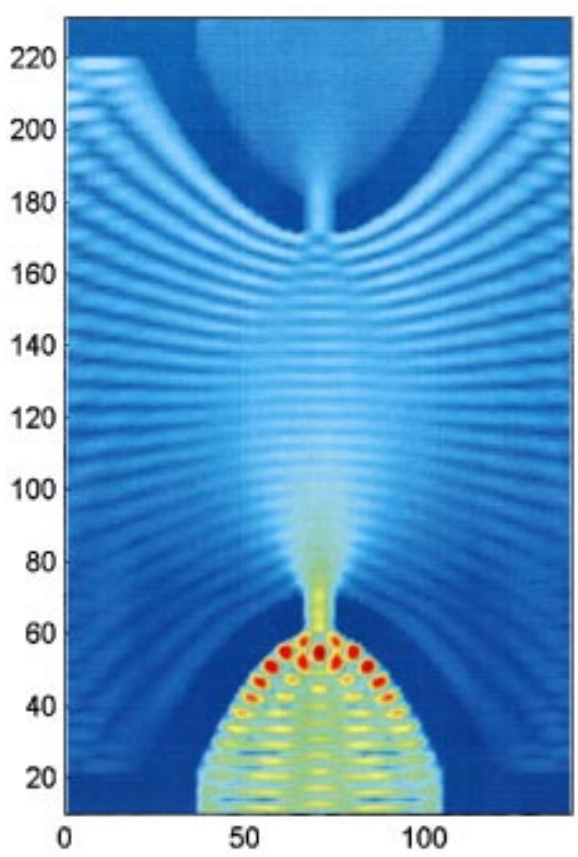

$\varepsilon=2500, v_{g}=1250$

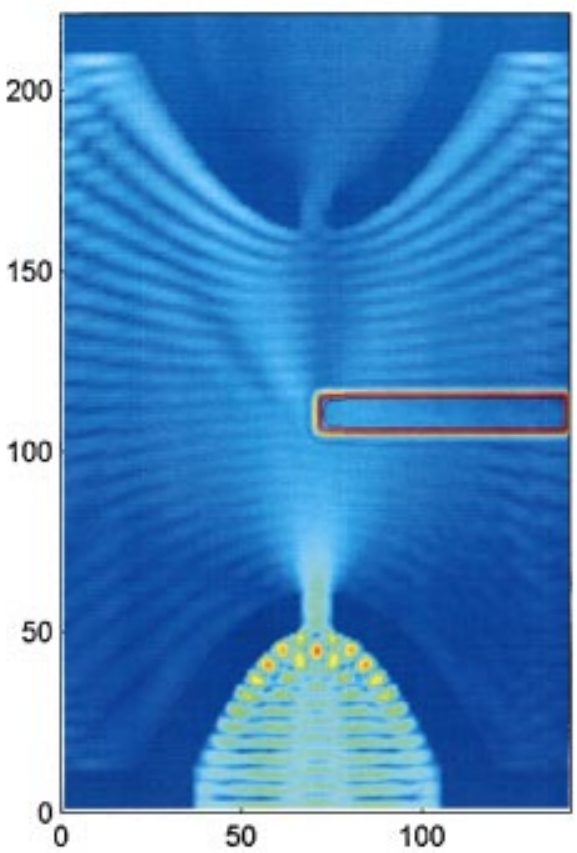

FIG. 4. (Color) The modulus of the wave-function for two gate voltages $V_{g}=0,1250$ for incident electron in the first subband of the emitter with energy $\epsilon=2500$.
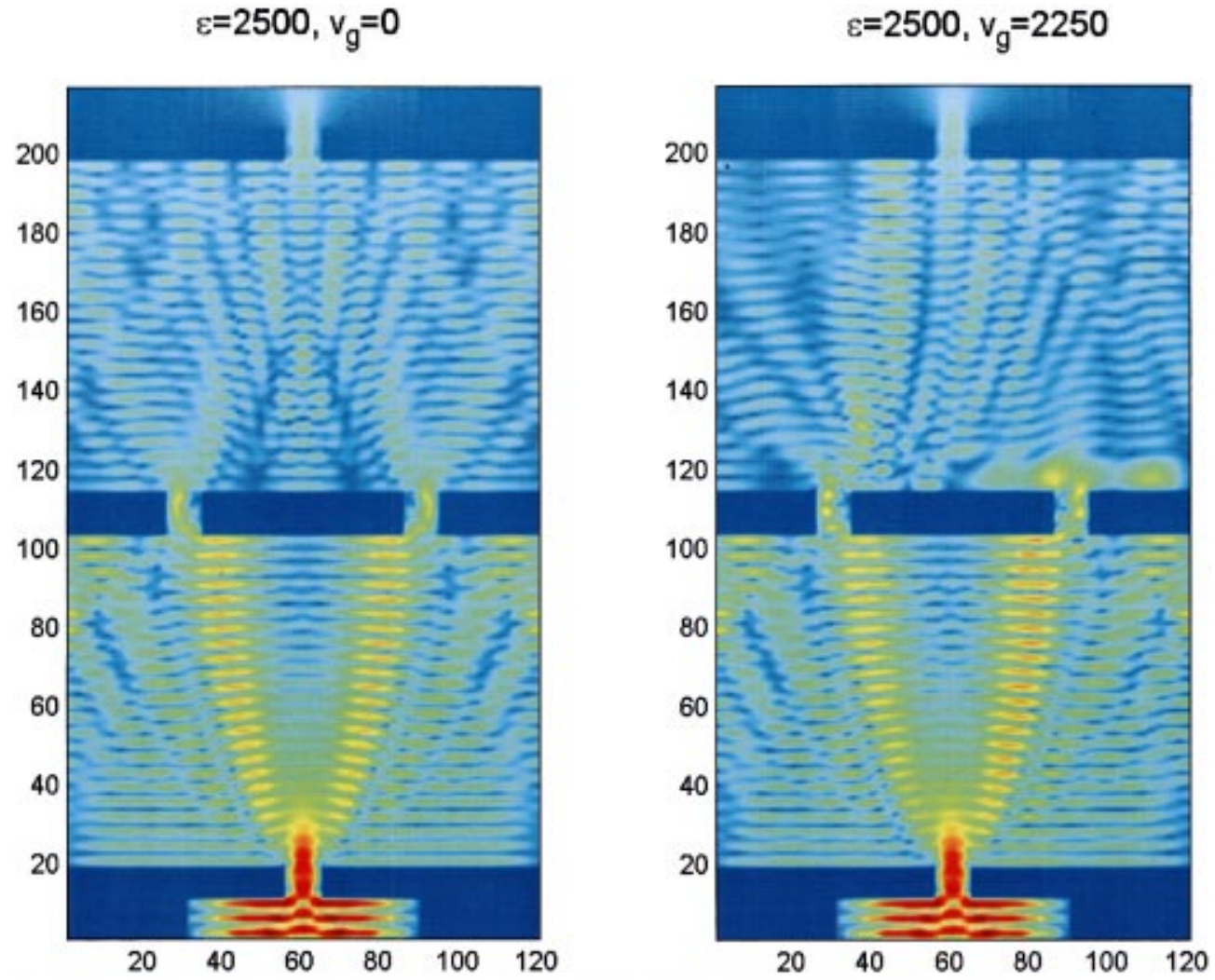

FIG. 8. (Color) The wave function for double-slit geometry at two gate voltages, $V_{g}=0$ and $V_{g}=2250$, the latter corresponding to the last significant maximum in the conductance vs gate-voltage characteristic of top panel of Fig. 7. 


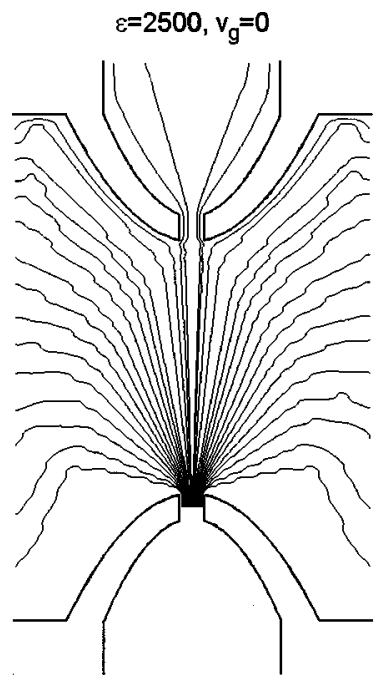

$\varepsilon=2500, v_{g}=1250$

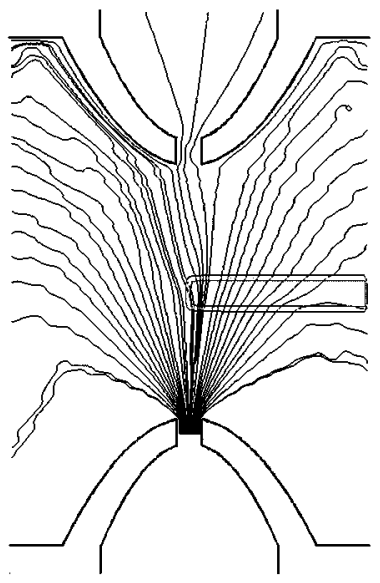

FIG. 5. Stream lines for the gate voltages of Fig. 4. The stream lines passing under the gate and ending in the collector are essentially straight lines and can be described by one-dimensional quantum mechanics.

$$
\theta=\frac{1}{\hbar} \int d y \sqrt{2 m^{*}[E-V(x, y, h)]} .
$$

Neglecting the paths that pass near the edge of the PMG we can take the $x \rightarrow \infty$ limit of Eq. (6), and find

$$
V(y, h)=\frac{V_{g}}{\pi}\left[\arctan \left(\frac{y+w / 2}{h}\right)-\arctan \left(\frac{w / 2-y}{h}\right)\right],
$$

which, to a good approximation, provided that $h \ll w$, can be approximated by a rectangular barrier of width $w$ and height $V_{g}$. The phase difference between electrons passing under the PMG and those that do not is then readily found to be

$$
\Delta \theta=w\left(\sqrt{\epsilon}-\sqrt{\epsilon-\mathrm{V}_{g}}\right) .
$$

(Again, all energies are in units of $E_{0}$.) This derivation provides a justification for the phenomenological expression for phase $\Delta \phi$ used in the experimental Refs. 2 and 3, see also Ref. 12. Maximal constructive interference occurs when $\Delta \theta=2 n \pi$, which leads to expected maxima for PMG biases at

$$
\mathrm{V}_{g}(n)=4 \pi \sqrt{\epsilon} n / w-(2 \pi n / w)^{2} .
$$

Figure 3 shows the numerically computed conductance vs PMG bias for incident electron energies $\epsilon=2500$ (corresponding to 16 propagating modes), and $\epsilon=5000$ (24 modes). The conductance was evaluated with the LandauerBüttiker multichannel formula: ${ }^{13,14}$

$$
G_{E C}=\frac{2 e^{2}}{h} T_{E C}=\frac{2 e^{2}}{h} \sum_{n m}\left|t_{n m}\right|^{2} \frac{k_{m}}{k_{n}} .
$$

The voltages at the base contacts are set to zero. The arrows in Fig. 3 indicate the predictions of the semiclassical formula, and one observes a good overall agreement. As could be expected, the conductance does not show any interference structure when the gate voltage becomes very large, because the effective potential due to the gate is then nontransmitting.

In order to gain a deeper understanding of why the onedimensional model works so well in this particular case it is useful to study the quantum-mechanical streamlines (for applications to several other physical systems one may consult, e.g., Refs. 15-19). We use the following construction. Writing the wave function in terms of an amplitude and a phase,

$$
\psi=\sqrt{\rho} \exp (i S / \hbar)
$$

the real and imaginary parts of the time-independent Schrödinger equation,

$$
\left[\frac{-\hbar^{2}}{2 m^{*}} \nabla^{2}+V\right] \psi=E \psi
$$
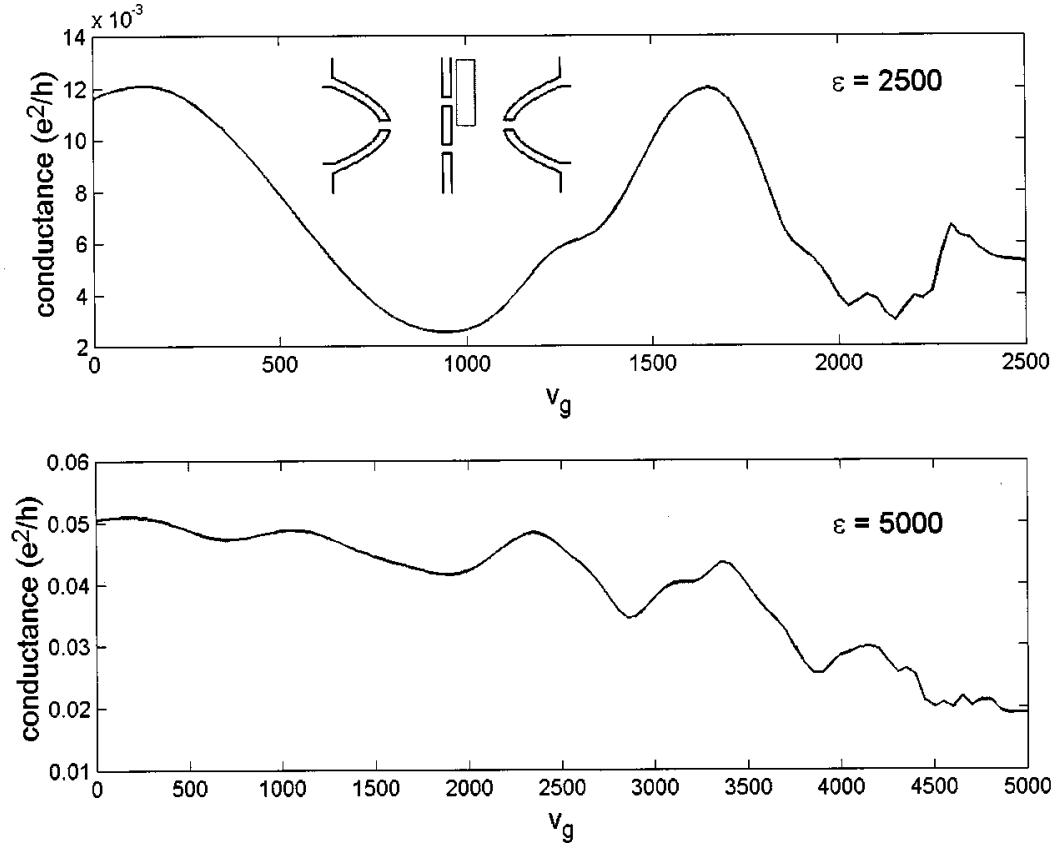

FIG. 6. Conductance vs gate voltage for double-slit geometry, with curved emitter and collector. The top view of the device is shown as an inset. 

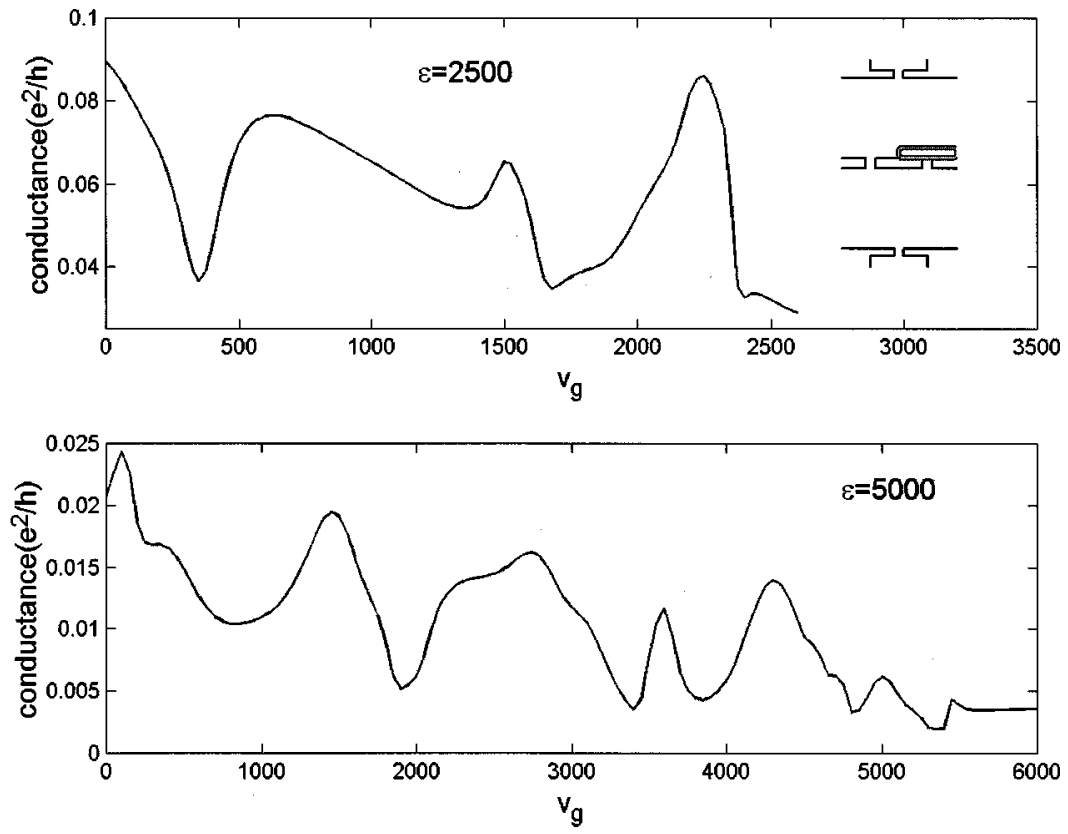

FIG. 7. Conductance vs gate voltage for double-slit geometry, with rectangular emitter and collector. A schematic view of the device is shown as an inset.

yield

$$
\begin{gathered}
\frac{1}{2} m^{*} \mathrm{v}^{2}+V+V_{Q M}=E, \\
\boldsymbol{\nabla} \cdot \mathbf{j}=0,
\end{gathered}
$$

where

$$
\begin{gathered}
\mathbf{j}=\frac{1}{m} \rho \boldsymbol{\nabla} S, \\
V_{Q M}=-\left(\frac{\hbar^{2}}{2 m}\right)\left[\frac{1}{2} \frac{\nabla^{2} \rho}{\rho}-\frac{1}{4} \frac{(\boldsymbol{\nabla} \rho)^{2}}{\rho^{2}}\right]=-\frac{\hbar^{2}}{2 m} \frac{\nabla^{2} \rho^{1 / 2}}{\rho^{1 / 2}} .
\end{gathered}
$$

According to Bohm, ${ }^{20}$ one interprets the electrons as "real" particles in the classical sense, following a continuous and causally defined trajectory with a well-defined position $\mathbf{x}$ with the momentum given by $m \dot{\mathbf{x}}=\boldsymbol{\nabla} S$. The force acting on the particle is not derivable from the classical potential $V$ alone, but acquires a quantum-mechanical contribution from $V_{Q M}$, Eq. (16). The current stream lines can then be computed as in classical mechanics but including the quantum force. The streamlines can be viewed as an alternative graphical presentation of the quantum-mechanical probability current density, see, e.g., Fig. 1 of Ref. 7.

Figure 4 shows the computed wave function for two different values of the gate potential $V_{g}$, the right panel corresponding to the first maximum in Fig. 3, top panel. We direct attention to the following features. (i) The wave functions display a rather regular pattern even at a finite gate voltage (which breaks the mirror symmetry of the problem). (ii) The curvilinear injector leads to a clear focusing effect. (iii) The effective wave length is clearly longer under the gate than elsewhere in the 2DEG, in accordance with the expectations. Figure 5 shows the computed stream lines. We note that most of the stream lines ending in the collector are, even in the case of a finite gate potential, essentially straight lines.
Thus they can be described by one-dimensional quantum mechanics, and consequently the semiclassical estimate for occurrence of conductance maxima, shown as arrows in Fig. 3 , works reasonably accurately. We next turn to the doubleslit geometry, where matters turn out to be quite different.

\section{DOUBLE SLIT GEOMETRY}

We now introduce the double slit. In order to compare most directly with the results obtained in the previous section, we first consider same emitters and collectors as before, even though the experiment was done with a different design (this will be discussed below). Figure 6 shows the computed conductance in the presence of the double slit, as indicated by the inset in the top panel. Again, we see oscillations in the conductance, however the values of the gate voltage at which the conductance is at maximum do not correspond to the values predicted by the simple estimates, such as Eq. (9). We
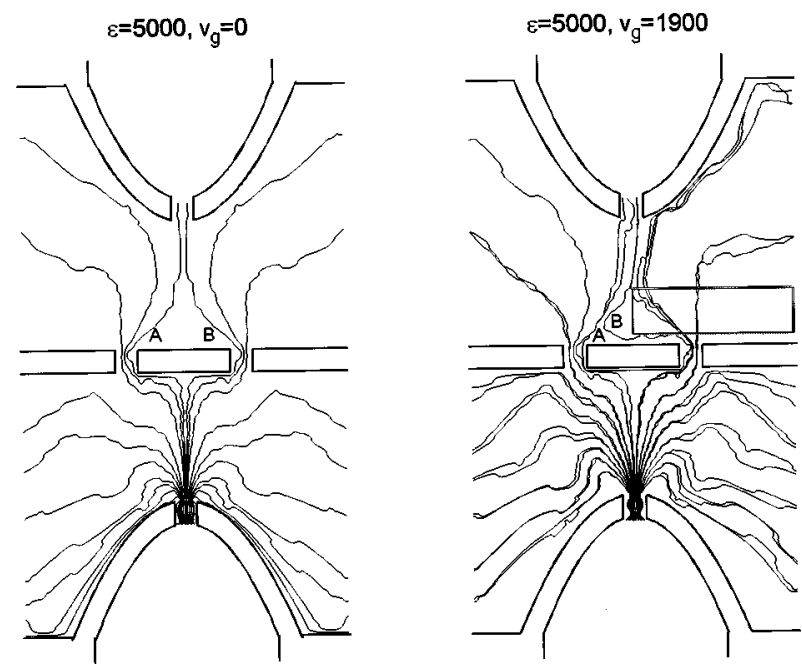

FIG. 9. Quantum stream lines for double-slit geometry with curved emitter and collector for gate voltages $V_{g}=0,1900$. 

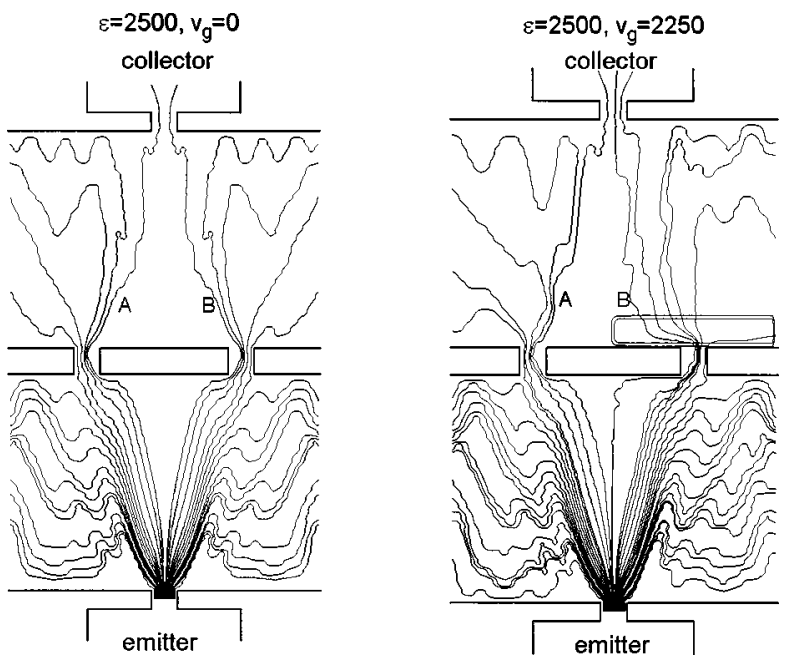

FIG. 10. Stream lines for double-slit geometry with rectangular emitter and collector.

next consider the experimental geometry of Ref. 3, where the emitter and collector are rectilinear. Figure 7 shows the computed conductance curves and Fig. 8 displays examples of computed wave functions. Conductance oscillations are quite evident in Fig. 7, but it is much harder to find any regular periodicity, in contrast to the curves for the device without the double slit of Fig. 3. Since our simulated conductance curves only have few maxima (because of the computational restrictions to relatively low energies) we did not find a Fourier-analysis helpful (as was the case for experiments which had a larger available gate potential range): the resulting spectrum is dominated by spurious edge effects. Finally, Figs. 9 and 10 show the computed stream lines for the curved and rectangular emitters/collectors, respectively. We draw attention to the qualitatively different picture as compared to the device without the double-slit: the approximately straight line form of the stream lines is almost entirely lost. Most importantly, the stream lines passing under the gate show a rather irregular structure with a very wide range of effective path lengths under the gate. It is instructive to consider the pair of paths denoted by $A$ and $B$ (which have symmetric initial conditions at the emitter): the combined effect of the double-slit and the gate is to distort $B$ significantly, and it is not surprising that a model based on pairs of straight-line trajectories fails to describe it properly. ${ }^{21}$

The device with rectangular emitter/collector, as in Ref. 3, has another intriguing property: there is a clear tendency to form resonances between the emitter and the double slit (and, to a lesser extent, between the double slit and the collector). The effect of these resonances can be understood in terms of a beating phenomenon: their frequency mixes with that due to the PMG, and in general one can expect much more irregular conductance vs gate-voltage curves, as is the case with curved emitters/collectors. It is quite conceivable that this mixing can contribute to the half-frequency oscillation observed in Ref. 3. Another indication of these "size resonances" is that the conductance is not always maximum at zero gate voltage (see, e.g., the lower panel of Fig. 7): this is because the emitter quantum point contact is not always matched to the resonator modes of the cavity formed by the gates defining the emitter and the double slit, and a finite gate voltage can move the resonator modes so as to achieve more efficient injection from the emitter. In view of our simulations it would appear to be interesting to repeat the double-slit experiment with curved emitter/collector: the experimental trace is expected to be easier to interpret because one achieves a better focused injection and diminishes complications due to the resonator modes.

\section{CONCLUSION}

We have presented simulations of phase-coherent charge transport in gated mesoscopic structures. The simulations can describe the experiments at least qualitatively, and under certain circumstances quantitatively. Using quantum stream lines as an interpretative tool we are able to offer an explanation of why certain experiments can be interpreted with the help of one-dimensional models, while others cannot. We find in general great sensitivity to geometric effects, however these can be controlled at least to some extent by careful device design aided with simulations of the kind presented here, in particular when extended to higher energies.

\section{ACKNOWLEDGMENTS}

This work was supported by INTAS-RFBR Grant No. 95-IN-RU-657 and RFFI Grant No. 97-02-16305. K.N.P. acknowledges INTAS Grant No. YSF 98-99, and A.F.S. thanks Mikroelektronik Centret for hospitality.
${ }^{1}$ For recent reviews, see Mesoscopic Phenomena in Solids, edited by B. L. Altshuler, P. A. Lee, and R. A. Webb (Elsevier, Amsterdam, 1991); Mesoscopic Electron Transport, Vol. 345 of NATO Advanced Study Institute, Series E: Applied Sciences, edited by L. L. Sohn, L. P. Kouwenhoven, and G. Schön (Kluwer, Dordrecht, 1997).

${ }^{2}$ A. Yacoby, U. Sivan, C. P. Umbach, and J. M. Hong, Phys. Rev. Lett. 66, 1938 (1991).

${ }^{3}$ A. Yacoby, M. Heiblum, V. Umansky, H. Shtrikman, and D. Mahalu, Phys. Rev. Lett. 73, 3149 (1994).

${ }^{4}$ C. Hodges, H. Smith, and J. W. Wilkins, Phys. Rev. B 4, 302 (1972); A. V. Chaplik, Zh. Éksp. Teor. Fiz. 60, 1845 (1971) [Sov. Phys. JETP 33, 997 (1971)]; G. F. Giuliani and J. J.
Quinn, Phys. Rev. B 26, 4421 (1982).

${ }^{5}$ Assuming that the gate potential gives rise to an effective square barrier of height $V_{\text {eff }}\left(V_{g}\right)$, the effective Fermi momentum under the gate is $k_{F}^{\prime}=k_{F} \sqrt{1-V_{\text {eff }}\left(V_{g}\right) / \epsilon_{F}}$. The expression used in the text is obtained by assuming that the effective barrier height scales linearly with the gate potential, and reaches the Fermi energy at gate voltage $V_{\text {dep }}: V_{\text {eff }}\left(V_{g}\right)=\epsilon_{F} V_{g} / V_{\text {dep }} ; V_{\text {dep }}$ can be extracted from experiment. In Sec. III, we show how the same expression emerges from a simplified solution of the Laplace equation.

${ }^{6}$ From the vast literature we quote but a few representative papers where further references can be found: F. Sols and M. Macucci, Phys. Rev. B 41, 11887 (1990); Y. Takagaki and D. K. Ferry, 
ibid. 44, 8399 (1991); A. Weisshaar, J. Lary, S. M. Goodnick, and V. K. Tripathi, J. Appl. Phys. 70, 355 (1991); K.-F. Berggreen and Zh.-L. Ji, Phys. Rev. B 47, 6390 (1993); M. Leng and C. S. Lent, Phys. Rev. Lett. 71, 137 (1993); N. Pichugin and A. F. Sadreev, Phys. Rev. B 56, 9662 (1997).

${ }^{7}$ P. Exner, P. Šba, A. F. Sadreev, P. Středa, and P. Feher, Phys. Rev. Lett. 80, 1710 (1998).

${ }^{8}$ T. Ando, Phys. Rev. B 44, 8017 (1991).

${ }^{9}$ Our typical simulations have a mesh of $200 \times 240$ sites, with a computational time of the order of $10 \mathrm{~h}$.

${ }^{10}$ J. H. Davies, I. A. Larkin, and E. V. Sukhorukov, J. Appl. Phys. 77, 4504 (1995).

${ }^{11}$ The potential for the semi-infinite phase-modulating gate is constructed as a superposition of two infinite rectangles, see Ref. 10 Eq. (3.9).

${ }^{12}$ It is, of course, quite straightforward to use the fully quantummechanical formula for the transmission coefficient over a square barrier to compute the phase difference; a numerical test shows that the two results are in very good agreement except for energies just above the barrier. Thus, the semiclassical formula used in the text is sufficient for present purposes.

${ }^{13}$ M. Büttiker, Phys. Rev. Lett. 57, 1761 (1986).

${ }^{14}$ S. Datta, Electronic Transport in Mesoscopic Systems (Cambridge University Press, Cambridge, 1997).

${ }^{15}$ J. O. Hirschfelder, A. C. Christoph, and W. E. Palke, J. Chem. Phys. 61, 5435 (1974); J. O. Hirschfelder, G. J. Goebel, and L. W. Bruch, ibid. 67, 5456 (1974); J. O. Hirschfelder, ibid. 67, 5477 (1977).
${ }^{16}$ H. Wu and D. W. L. Sprung, Phys. Lett. A 183, 413 (1993).

${ }^{17}$ T. Lundberg, Ph.D. Thesis, Linköping University, 1998.

${ }^{18}$ C. R. Leavens and G. C. Aers, Scanning Tunneling Microscopy III, Springer Series in Surface Science Vol. 29 (Springer, Berlin, 1993), p. 105.

${ }^{19}$ J. R. Barker, p. 171 in Quantum Transport in Ultrasmall Devices, edited by D. K. Ferry, H. L. Grubin, C. Jacoboni, and A.-P. Jauho, Vol. 342 of NATO Advanced Study Institute Series B: Physics (Plenum Press, New York, 1995).

${ }^{20}$ D. Bohm, Phys. Rev. 85, 166 (1952); 85, 180 (1952); C. Dewdney and B. J. Hiley, Found. Phys. 12, 27 (1982); P. Holland, The Quantum Theory of Motion (Cambridge University Press, Cambridge, 1993); D. Bohm and B. J. Hiley, The Undivided Universe: an Ontological Interpretation of Quantum Mechanics (Routledge, London, 1994).

${ }^{21}$ The simple formula Eq. (8) is a special case of the semiclassical phase formula $\theta \propto \int d \mathbf{l} \cdot \mathbf{p}$, where the line integral is calculated along a given trajectory. In the double-slit geometry small changes in the initial conditions lead to large changes in the trajectory passing under the gate, and it hence follows that the interference condition obtained by comparing pairs of trajectories is a strongly fluctuating function. Consequently, the conductance, which is a sum of all trajectories joining the emitter and collector, cannot be a simple function of the width and/or the potential of the phase-modulating gate. The situation is quite different without the double slit, Fig. 5, where the trajectories are stable functions of the initial conditions, and hence the simple formula works rather well, Fig. 3. 